\title{
Optical Study of the Electronic Structure and Magnetic Ordering in a Weak Ferromagnet $\mathrm{FeBO}_{3}$
}

\author{
P. A. Markovin ${ }^{a}$, A. M. Kalashnikova ${ }^{a, b}$, R. V. Pisarev ${ }^{a}$, and Th. Rasing ${ }^{b}$ \\ ${ }^{a}$ Ioffe Physicotechnical Institute, Russian Academy of Sciences, St. Petersburg, 194021 Russia \\ e-mail: pisarev@mail.ioffe.ru \\ ${ }^{b}$ IMM, Radboud University Nijmegen, 6525ED Nijmegen, The Netherlands \\ Received October 15, 2007
}

\begin{abstract}
Spectral dependences of the relative permittivity $\varepsilon=\varepsilon_{1}-i \varepsilon_{2}$ of a uniaxial weak ferromagnet $\mathrm{FeBO}_{3}$ are measured for two principal polarizations in the energy range $0.6-5.6 \mathrm{eV}$. The positions have been determined for the charge-transfer transitions that make the main contribution to absorption above the bandgap and determine the refractive-index dispersion below the bandgap. The isotropic magnetic contribution to the refractive index has been detected by studying the temperature dependence of the refractive index in the range 100-700 K; its value $\left(\approx 2 \times 10^{-2}\right)$ is found to be record high for magnetic dielectrics. The energy shift of the effective oscillator, which characterizes the shift of the positions of the charge-transfer transitions due to magnetic ordering, is determined from these data within the framework of a single-oscillator model. The value of the exchange striction in $\mathrm{FeBO}_{3}$ is determined from optical measurements.
\end{abstract}

PACS numbers: 75.50.Ee, 78.20.-e, 78.20.Ci, 78.20.Ls

DOI: $10.1134 / \mathrm{S} 0021364007230063$

\section{INTRODUCTION}

Magnetically ordered materials and structures exhibit specific features in the spectra of electronic, magnon, and phonon excitations, because of which their interaction with electromagnetic radiation results in a number of physical phenomena. The same spectral features result in large values of linear and nonlinear magneto-optical phenomena such as Faraday and Kerr effects [1-3], magnetic linear birefringence [4, 5], and the generation of the second and higher optical harmonics [6]. These and other magneto-optical phenomena serve as an alternative and, sometimes, preferable method for studying electronic and magnetic structures.

In very recent years, a new area has been formed in the physics of magnetic materials whose main task is to study the processes of control of the magnetic state of solids using short laser pulses [7, 8]. Magneto-optical effects are the most effective and relatively simple means of monitoring ultrafast demagnetization processes [9], the excitation of spin precession [8], and magnetization reversal [10], when the large value of the former provides the possibility of achieving a high sensitivity and time resolution. This circumstance has put forward the problem of a more detailed spectroscopic investigation of the electronic structure as the microscopic basis of magneto-optical effects.

In this work, we report the results of studying the electronic structure and magnetic ordering in a wellknown weak ferromagnet iron borate $\mathrm{FeBO}_{3}$. A review of its magnetic, optical, and magneto-optical properties can be found in [11]; therefore, we will consider here only its brief characteristics. $\mathrm{FeBO}_{3}$ is crystallized in the rhombohedral calcite structure with the space group $D_{3 d}^{6}$ and, hence, is optically uniaxial. It represents a rare example of a magnetically ordered ferric oxide with a high transparency in the visible spectral region and with a Faraday rotation reaching $5000 \% \mathrm{~cm}$ [12]. From the magnetic point of view, $\mathrm{FeBO}_{3}$ is a two-sublattice easy-plane antiferromagnet with a weak in-plane moment and the Néel temperature $T_{N}=348 \mathrm{~K}$.

\section{EXPERIMENTAL PROCEDURES AND SAMPLES}

Studies of the dispersion of the dielectric functions $\varepsilon_{x x}$ and $\varepsilon_{z z}$ in the optical region $0.6-5.6 \mathrm{eV}$ were carried out using a spectroscopic ellipsometer in reflection geometry. The details of the studies and the corresponding calculations were described previously in [13]. The absorption spectrum $\alpha_{x x}$ in the region of relative transparency $1-3 \mathrm{eV}$ was obtained by measuring absorption in the transmission geometry.

The temperature dependence of the refractive index was studied using a homodyne interferometer at a helium-neon laser wavelength of $1.078 \mathrm{eV}(1.15 \mu \mathrm{m})$ [14-16]. A distinctive feature of this method is that the sensitivity of measurements is not reduced both under a strong (by 1-2 orders of magnitude) change in the intensity due to temperature variations of absorption 


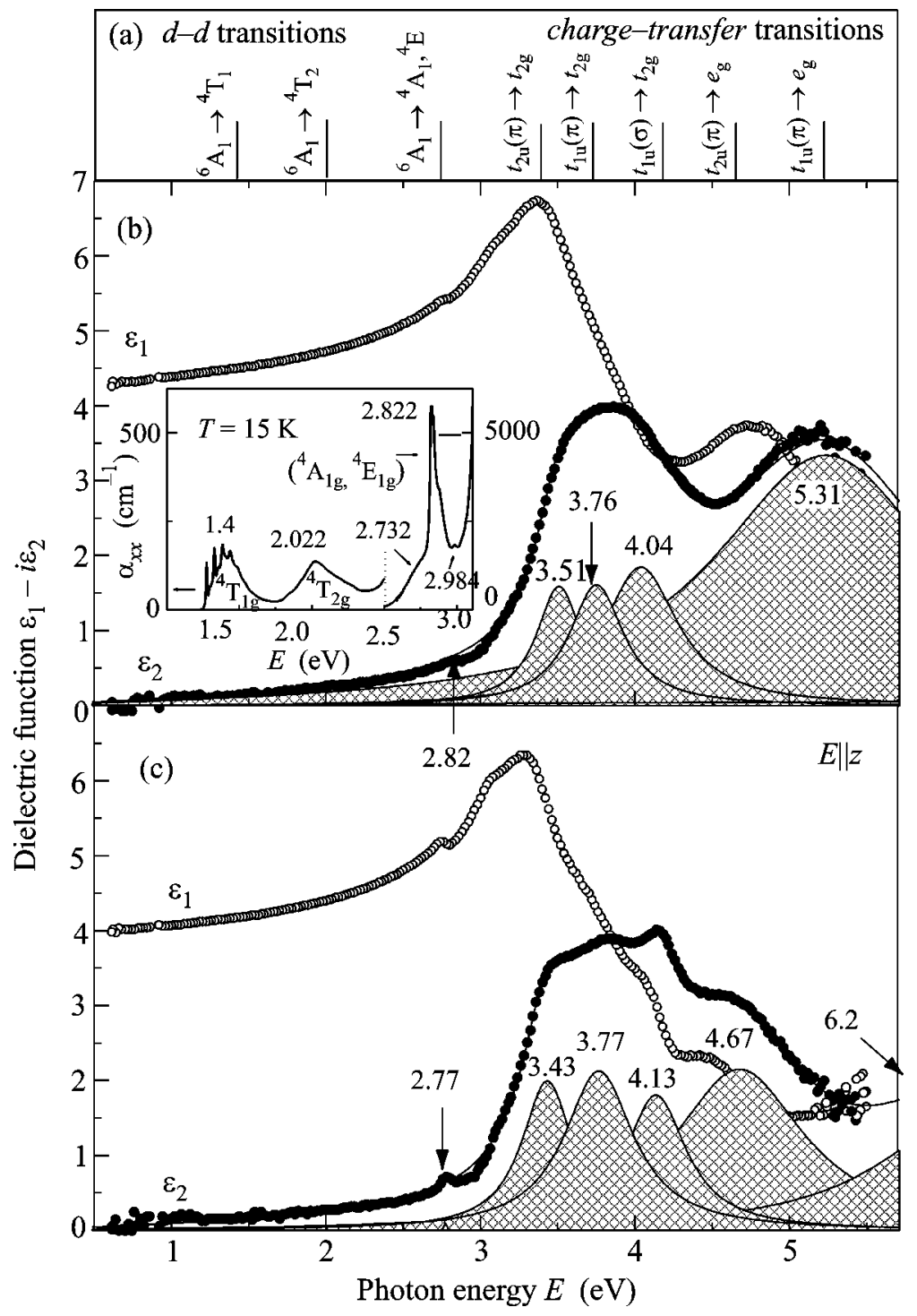

Fig. 1. (a) Scheme of localized $d-d$ and charge-transfer transitions according to [22]. (b and c) Spectral dependences of the real $\varepsilon_{1}$ ( $\circ$ ) and imaginary $\varepsilon_{2}(\bullet)$ parts of the relative permittivity for light polarized (b) perpendicularly to and (c) along the optical $z$ axis. Dotted lines correspond to the decomposition of the $\varepsilon_{2}$ spectrum using Eq. (2). The inset presents spectral dependences of the absorption index in the range of $d-d$ transitions [19].

and under light depolarization, for example, in the case of a domain structure formation. The relative variations of the optical light path in the crystal $\delta \psi(T)$ (relative to vacuum) normalized to the crystal thickness associated with variations of the refractive index $\delta n(T)$ and the thermal expansion of the sample $\delta l / l$ can be calculated by this method according to the equation

$$
\delta \psi_{i(j)}=\delta n_{i}+\left(n_{i}-1\right) \frac{\delta l_{j}}{l_{j}}
$$

where the first index $(i)$ indicates the light polarization and the second one $(j)$ indicates the propagation direction.
Samples were polished plane-parallel single-crystalline plates with thicknesses in the range 20$1000 \mu \mathrm{m}$. Plates with the normal oriented along and perpendicular to the optical $z$ axis were obtained from crystals grown from a solution in a melt and from a gas phase, respectively. This allowed the dielectric function of $\mathrm{FeBO}_{3}$ to be obtained for two main light polarizations.

\section{OPTICAL PROPERTIES OF $\mathrm{FeBO}_{3}$}

Spectra of the dielectric functions $\varepsilon_{z z}$ and $\varepsilon_{x x}$ for light polarized along and perpendicular to optical $z$ axis are given in Figs. 1b and 1c. The inset in Fig. 1 shows the absorption spectrum $\alpha_{x x}$ in the energy range $1-3 \mathrm{eV}$ for 


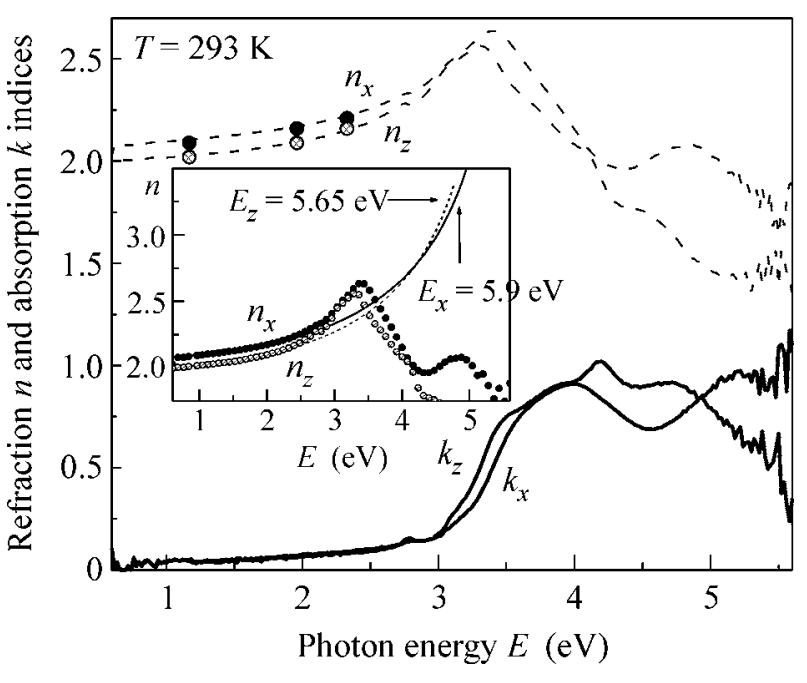

Fig. 2. Spectral dependences of the (dashed lines) refractive indices $n_{x}$ and $n_{z}$ and (solid lines) absorption indices $k_{x}$ and $k_{z}$. Signs $\bullet$ present data from [12]. The inset presents (symbols) the dispersion of the refractive indices $n_{x}$ and $n_{z}$ and (lines) its approximation using an effective oscillator [Eq. (3)]. Numbers are the energies of the corresponding oscillators.

light propagating along optical $z$ axis. Two groups of bands can be distinguished in the spectra. The first, less intense group is well distinguished only in the absorption spectrum $\alpha_{x x}$. It is presented by three bands below $3 \mathrm{eV}$ and corresponds to parity- and spin-forbidden transitions from the ground-state ${ }^{6} A_{1 g}$ level of the $\mathrm{Fe}^{3+}$ iron ion in a cubic crystal field to the ${ }^{4} T_{1 g},{ }^{4} T_{2 g}$, and $\left({ }^{4} A_{1 g},{ }^{4} E_{g}\right)$ levels. These bands were studied previously in [17-21]. The second group of several intense absorption bands above $3 \mathrm{eV}$ corresponds to allowed electricdipole charge-transfer transitions. A scheme of the corresponding transitions calculated in the cubic-field approximation for an iron ion in an octahedral cluster of oxygen ions [22] is given in Fig. 1a.

In Figs. $1 \mathrm{~b}$ and $1 \mathrm{c}$, it is seen that the intensity of the ${ }^{6} A_{1 g} \longrightarrow\left({ }^{4} A_{1 g},{ }^{4} E_{g}\right)$ transition is substantially higher than that of the two other forbidden transitions and that it is even distinguished against the background of allowed charge-transfer transitions. A calculation of the energy structure and optical spectra of $\mathrm{FeBO}_{3}$ in the framework of a model combining a one-electron description of the $s p$ states of boron and oxygen and a many-electron description of $d$ states of iron is presented in [20]. In this work, it was shown that the high intensity of the ${ }^{6} A_{1 g} \longrightarrow\left({ }^{4} A_{1 g},{ }^{4} E_{g}\right)$ band at an energy of $2.8 \mathrm{eV}$ is explained by a substantial contribution of charge-transfer transitions to its formation. From the presented spectral dependence $\alpha_{x x}$, it is seen that the intensity of the $2.8 \mathrm{eV}$ band at its maximum equals $\sim 6 \times 10^{3} \mathrm{~cm}^{-1}$, which is $\sim 30$ times higher than the maximum peak intensity of the two other bands caused by only $d-d$ transitions. The data published in $[17,20]$ give a value of about $3.1 \times 10^{3} \mathrm{~cm}^{-1}$, which is almost two times lower than our data. On the other hand, the intensities of the 1.4 and $2.0 \mathrm{eV}$ bands are close. Note that the spectrum of the absorption factor $\alpha_{x x}$ was obtained at $T=15 \mathrm{~K}$, while the spectra reported in $[17,20]$ were measured at a temperature of about $80 \mathrm{~K}$.

The ${ }^{6} A_{1 g} \longrightarrow\left({ }^{4} A_{1 g},{ }^{4} E_{g}\right)$ band is hybridized with charge-transfer transitions and exhibits a significant anisotropy (Figs. 1b and 1c). The local maximum in the $\varepsilon_{2 x x}$ spectrum is located higher in energy and has a lower amplitude than that in the $\varepsilon_{2 z z}$ spectrum. This is probably associated with the anisotropy of the chargetransfer transitions, which will be discussed below.

The bands in the absorption spectrum $\varepsilon_{2}$ (Figs. $1 b$ and 1c) located above $3 \mathrm{eV}$ correspond to the chargetransfer transitions. The fundamental absorption band edge is determined by the $p^{6} d^{5} \longrightarrow p^{5} d^{6}$ charge-transfer transition from the top of the valence band to the unfilled $d$ band of iron. The fundamental absorption edge for $\mathrm{FeBO}_{3}$ is conventionally determined at a level of $2.9 \mathrm{eV}[17,20]$, and theoretical calculations give a close value $[20,23]$. There are no ab initio electronic structure calculations for the above-bandgap absorption available in the literature.

The decomposition of the spectra into Lorentzian oscillators was performed by the equation

$$
\varepsilon=\varepsilon_{1}-i \varepsilon_{2}=\varepsilon_{0}+b E+\sum \frac{f_{0 j}}{E_{j}^{2}-E^{2}-i E \gamma_{j}},
$$

where $f_{0 j}$ is the oscillator strength for the $j$ th transition, $E_{j}$ is the central frequency of the transition, and $\gamma_{j}$ is a value characterizing the width of the transition. The term $b E$ takes into account the transitions located at higher energies outside the studied range. The calculation in the cubic-field approximation for an iron ion placed in an octahedral cluster of oxygen ions [22] allows the observed bands to be qualitatively compared with the charge-transfer transitions from sublevels of the valence band formed by the oxygen $s$ and $p$ states [23] to sublevels of the iron ion. It is seen in Figs. 1b and $1 \mathrm{c}$ that the anisotropy of the optical response of $\mathrm{FeBO}_{3}$ is determined by (a) the anisotropy of the transitions lying in the range $4.6-5.5 \mathrm{eV}$ and (b) the anisotropy of the lowest $t_{1 u}(\sigma) \longrightarrow t_{2 g}$ transition. It is the second factor that determines the anisotropy of the $2.8 \mathrm{eV}$ band and the anisotropy of the fundamental absorption edge. It is clearly seen in Fig. 2, where the spectra of absorption factor $k$ and refractive index $n$ for light polarized perpendicular and parallel to the $z$ axis are shown.

The dispersion and anisotropy of the refractive index (Fig. 2) in the range $1-2 \mathrm{eV}$ are also determined by the charge-transfer transitions located above $3 \mathrm{eV}$ and their anisotropy. With accuracy to experimental 


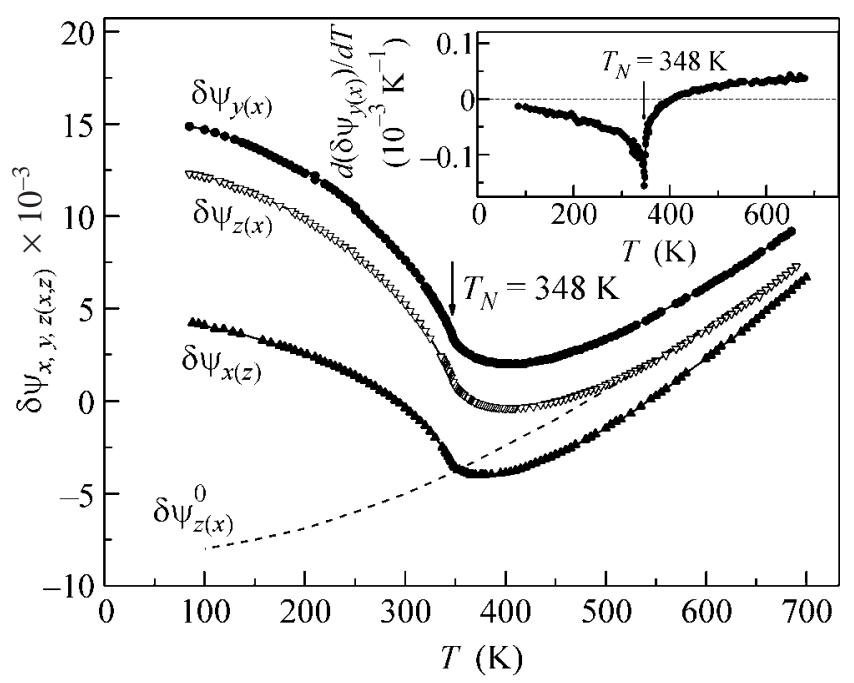

Fig. 3. Temperature dependences of the variation of the optical path $\delta \psi(T)$ for different light polarizations and propagation directions [see Eq. (1)]. The line gives an extrapolation of the regular contribution $\delta \psi_{z(x)}^{0}(T)$ to $\delta \psi_{z(x)}(T)$. The inset shows the temperature dependences of the derivative $\delta \psi_{y(x)}(T) / d T$.

data, the dispersion can be described by the one-oscillator equation

$$
n_{x, z}^{2}-1=\frac{f_{x, z}}{E_{x, z}^{2}-E^{2}},
$$

where $f_{x, z}$ is the effective oscillator strength, $E_{x, z}$ is the effective oscillator energy, and $E$ is the energy of the probing light (the inset in Fig. 2). From our experimental data, we determined the following values: $f_{z}=$ $95.3 \mathrm{eV}^{2}, E_{z}=5.65 \mathrm{eV}, f_{x}=114.7 \mathrm{eV}^{2}$, and $E_{x}=5.90 \mathrm{eV}$ for light polarized along and perpendicular to the $z$ axis, respectively. The accuracy of the one-oscillator approximation for the description of the dispersion of the refractive index in the range $1-2 \mathrm{eV}$ is, with regard to experimental errors, $\delta n \approx 0.002$.

\section{MAGNETIC REFRACTION OF LIGHT}

Figure 3 presents variations in the relative optical paths $\delta \psi_{x(z)}(T), \delta \psi_{y(x)}(T)$, and $\delta \psi_{z(x)}(T)$ for different light polarizations and propagation directions. Upon the transition from the magnetic to paramagnetic phase, the temperature dependence $\delta \psi(T)$ exhibits a welldefined feature (see inset in Fig. 3). As follows from Eq. (1), to determine the genuine magnetic contribution to the variations of the refractive index $(\delta n)$, it is necessary to distinguish the contribution due to the thermal expansion of the sample in the dependences in Fig. 3. Although there are no data in the literature on the thermal expansion coefficients, the estimates given below show that one may assume that $\delta \psi=\delta n$ for iron borate in the temperature range 100-700 K with an accuracy

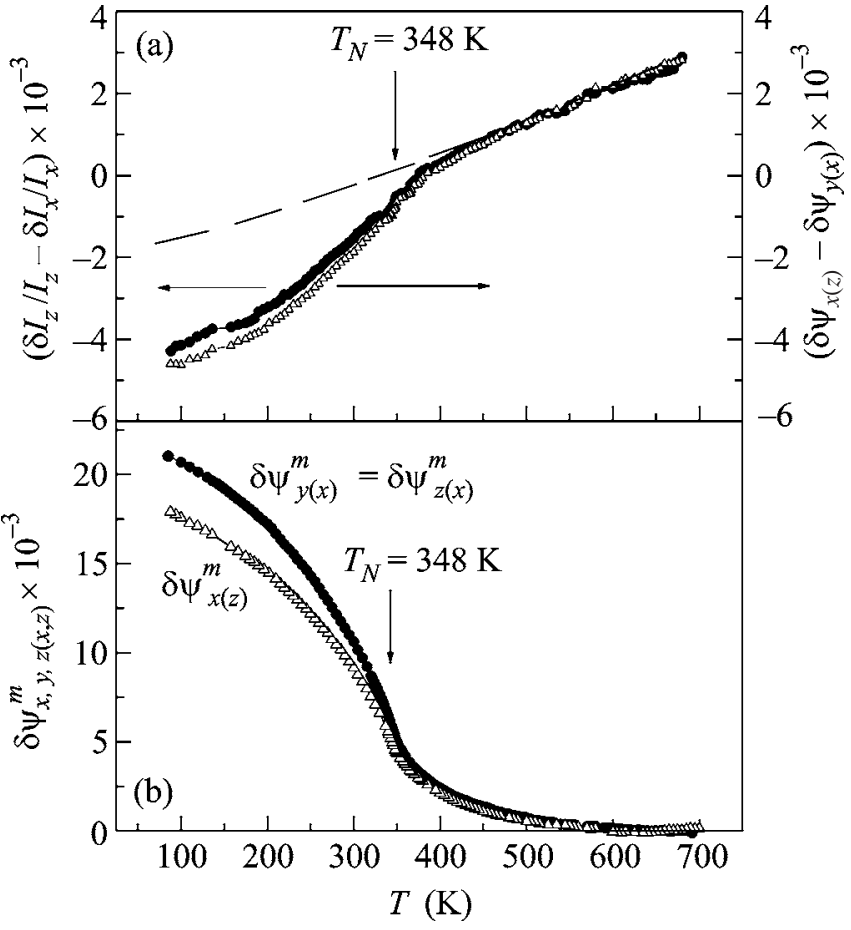

Fig. 4. (a) Temperature dependences $\left(\delta l_{z}(T) / l_{z}-\delta l_{x}(T) / l_{x}\right) \approx$ $\delta l_{z}(T) / l_{z}(\bullet)$ and $\delta \psi_{x(z)}-\delta \psi_{y(x)}(\triangle)$. The line gives an extrapolation of the regular contribution to the thermal expansion $\delta l_{z}(T) / l_{z}$. (b) Temperature dependences of the magnetic contributions to the light refraction $\delta n_{x, z}^{m} \approx$ $\delta \psi_{y(x), z(x)}^{m}(T)$. The difference between the $\delta \psi_{x(z)}^{m}(T)$ and $\delta \psi_{y(x)}^{m}(T)$ is associated with the difference between the contributions of the thermal expansion and the exchange magnetostriction along the $x$ and $z$ axes.

of about $10 \%$. In the calculations, we used our data for the principal values of the refractive index (Fig. 2) for a photon energy of $1.078 \mathrm{eV} n_{x}=n_{y}=2.101$ and $n_{z}=$ 2.022 .

The difference in the values $\delta \psi_{y(x)}(T)-\delta \psi_{z(x)}(T)$ measured upon light passing along and perpendicular to the optical axis allows the difference between the thermal expansions $\delta l_{z}(T) / l_{z}-\delta l_{x}(T) / l_{x}=\left(\delta \psi_{x(z)}(T)-\right.$ $\left.\delta \psi_{y(x)}(T)\right) /\left(n_{x}-1\right)$ along the $z$ and $x$ axes to be determined. Figure 4 a presents the experimental temperature dependence $\delta \psi_{x(z)}(T)-\delta \psi_{y(x)}(T)$ and the calculated dependence $\delta l_{z}(T) / l_{z}-\delta l_{x}(T) / l_{x}$. The thermal expansion of $\mathrm{FeBO}_{3}$ perpendicular to the $z$ axis in the range 80$500 \mathrm{~K}$ does not exceed values $\delta l_{x}(T) / l_{x} \leq 5 \times 10^{-4}$ in relative units [24]. From our calculations, it follows that, in the same temperature range, $\delta l_{z}(T) / l_{z}-\delta l_{x}(T) / l_{x} \approx 5 \times$ $10^{-3}$ (Fig. 4a); therefore, it may be assumed for $\mathrm{FeBO}_{3}$ that $\delta l_{z}(T) / l_{z}-\delta l_{x}(T) / l_{x} \approx \delta l_{z}(T) / l_{z}$ with an accuracy of the order of $10 \%$. A quantitative comparison shows that the contribution of the thermal expansion to $\delta \psi_{y(x)}(T)$ and $\delta \psi_{z(x)}(T)$ is lower than the variations in these values 
by an order of magnitude. Thus, the temperature dependences $\delta \psi_{y(x)}(T)$ and $\delta \psi_{z(x)}(T)$ presented in Fig. 3 correspond to the temperature variations of the principal values of the refractive index $\delta \psi_{y(x)}(T) \approx \delta n_{x}(T) \approx \delta n_{y}(T)$, $\delta \psi_{z(x)}(T) \approx \delta n_{z}$.

To quantitatively estimate the value and the temperature dependence of the magnetic contribution $\delta n^{m}$ to the refractive index, it is necessary to distinguish the component not associated with magnetic ordering in the temperature dependences of the light refraction (Fig. 3); that is, the regular crystallographic part of the temperature dependence $\delta n^{0}(T) \approx \delta \psi^{0}(T)$. As was shown in $[14,25]$, the dependences $\delta n_{x, z}^{0}(T)$ can be approximated by the corresponding integral DebyeEinstein function. We used this approach to approximate the temperature variations of the regular part of the light refraction in $\mathrm{FeBO}_{3}$. Figure 3 demonstrates an extrapolation of the regular part $\psi_{z(x)}^{0}(T)$ of the temperature variations of the light refraction in $\mathrm{FeBO}_{3}$ from the region of high temperatures, where one may neglect the effect of magnetic ordering. The difference between the experimental curves $\psi(T)$ and the dependences $\psi^{0}(T)$ represents the total magnetic contribution to the light refraction for the ordinary and extraordinary rays, which is shown in Fig. 4b. This contribution equals $\delta n_{x}^{m} \approx \delta n_{z}^{m} \approx\left(\delta \psi_{y, z(x)}^{m}\right) \approx 2 \times 10^{-2}$ at $80 \mathrm{~K}$. This value of the magnetic contribution to the refractive index is a record for magnetic dielectrics and is inferior only to the analogous value in magnetic semiconductors [5]. The magnetic contribution to the principal values of the refractive index found in this work exceeds the value of the magnetic linear light birefringence by two orders of magnitude; the latter is determined by anisotropic increments in the refraction depending upon the orientation of the magnetic moments with respect to the crystallographic axes. Hence, it follows that the found contribution is isotropic with respect to magnetization.

From our optical data, we can also estimate the exchange striction along the $z$ axis. The dashed line in Fig. 4a shows an extrapolation of the regular contribution not associated with the magnetic order to the thermal expansion along the $z$ axis (performed using the same procedure as for the light refraction). The difference between the dashed line and the curve designated by dots represents the contribution of the exchange striction along the $z$ axis, which reaches a value of $\approx 2 \times 10^{-3}$ at $100 \mathrm{~K}$.

From the microscopic point of view, the magnetic contribution to the light refraction is associated with the isotropic exchange interaction $[14,5]$. From our data on the determination of $\delta n_{x}^{m}$ and $\delta n_{z}^{m}$ at $100 \mathrm{~K}$, the energy shift of the effective oscillator $\delta E_{x, z}^{m}$ can be estimated in the framework of the one-oscillator model under the assumption that the effective oscillator strength does not change substantially upon magnetic ordering

$$
\delta n_{x, z}^{m}=\frac{n_{x, z}^{2}-1}{2 n_{x, z}}\left(-\frac{2 E_{x, z}^{2}}{E_{x, z}^{2}-E^{2}}\right) \frac{\delta E_{x, z}^{m}}{E_{x, z}},
$$

$\delta E_{x}^{m} \approx-6.9 \times 10^{-2} \mathrm{eV}(1.2 \%)$ and $\delta E_{z}^{m} \approx-7.3 \times$ $10^{-2} \mathrm{eV}(1.3 \%)$ at $T=100 \mathrm{~K}$. Because the effective oscillator energy lies in the region of the charge-transfer transitions, the energy shift of the effective oscillator characterizes the shift of these transitions due to an isotropic exchange.

We are grateful to V.V. Pavlov for help in the treatment of the results, to B.B. Krichevtsov for familiarization with birefringence data, and to A.F. van Etteger and A.J. Toonen for technical assistance. This work was supported by the Russian Federal Agency on Science and Innovations, the Russian Foundation for Basic Research, the Presidium and the Branch of Physical Sciences of the Russian Academy of Sciences, the Dutch Nanotechnology Network NanoNed, de Nederlandse Organisatie voor Wetenschappelijk Onderzoek, and INTAS.

\section{REFERENCES}

1. V. V. Eremenko, N. F. Kharchenko, Yu. G. Litvinenko, and V. M. Naumenko, Magneto-Optics and Spectroscopy of Antiferromagnetics (Naukova Dumka, Kiev, 1989; Springer, New York, 1992).

2. A. K. Zvezdin and V. A. Kotov, Modern Magnetooptics and Magnetooptical Materials (IOP, Bristol, 1997).

3. Magneto-Optics, Ed. by S. Sugano and N. Kojima (Springer, Berlin, 2000).

4. G. A. Smolenskiŭ, R. V. Pisarev, and I. G. Siniǔ, Usp. Fiz. Nauk 116, 231 (1975) [Sov. Phys. Usp. 18, 410 (1975)].

5. J. Ferré and G. A. Gehring, Rep. Prog. Phys. 47, 513 (1984).

6. M. Fiebig, V. V. Pavlov, and R. V. Pisarev, J. Opt. Soc. Am. B 22, 96 (2005).

7. Spin Dynamics in Confined Magnetic Structures, Ed. by B. Hillebrands et al. (Springer, Berlin, 2002, 2003, 2006), Vols. 1, 2, 3.

8. A. V. Kimel, A. Kirilyuk, F. Hansteen, et al., J. Phys. D: Condens. Matter 19, 043201 (2007).

9. E. Beaurepaire, J.-C. Merle, A. Daunois, and J.-Y. Bigot, Phys. Rev. Lett. 76, 4250 (1996).

10. C. D. Stanciu, F. Hansteen, A. V. Kimel, et al., Phys. Rev. Lett. 99, 047601 (2007).

11. Landolt-Börnstein, Numerical Data and Functional Relationships in Science and Technology, Ed. by H. P. J. Wijn (Springer, Berlin, 1993), Group III, Vol. 27h.

12. R. Wolfe, A. J. Kurtzig, and R. C. LeCraw, J. Appl. Phys. 41, 1218 (1970).

13. A. M. Kalashnikova and R. V. Pisarev, Pis'ma Zh. Éksp. Teor. Fiz. 78, 175 (2003) [JETP Lett. 78, 143 (2003)]. 
14. P. A. Markovin and R. V. Pisarev, Zh. Éksp. Teor. Fiz. 77, 2461 (1979) [Sov. Phys. JETP 50, 1190 (1979)].

15. R. V. Pisarev, B. B. Krichevtsov, P. A. Markovin, et al., Phys. Rev. B 28, 2677 (1983).

16. B. B. Krichevtsov, P. A. Markovin, S. V. Petrov, and R. V. Pisarev, Zh. Eksp. Teor. Fiz. 86, 2262 (1984) [Sov. Phys. JETP 59, 1316 (1984)].

17. I. S. Édel'man, A. V. Malakhovskiǔ, T. I. Vasil'eva, and V. N. Seleznev, Fiz. Tverd. Tela (Leningrad) 14, 2810 (1972) [Sov. Phys. Solid State 14, 2442 (1972)]; V. N. Zabluda, A. V. Malakhovskiǔ, and I. S. Édel'man, Fiz. Tverd. Tela (Leningrad) 27, 133 (1985) [Sov. Phys. Solid State 27, 77 (1985)].

18. B. Andlauer, O. F. Schirmer, and J. Schneider, Solid State Commun. 13, 1655 (1973).

19. A. M. Kalashnikova, R. V. Pisarev, I. N. Bezmaternykh, et al., Pis'ma Zh. Éksp. Teor. Fiz. 81, 568 (2005) [JETP Lett. 81, 452 (2005)].
20. S. G. Ovchinnikov and V. N. Zabluda, Zh. Éksp. Teor. Fiz. 125, 150 (2004) [JETP 98, 135 (2004)].

21. A. G. Gavrilyuk, I. A. Troyan, S. G. Ovchinnikov, et al., Zh. Éksp. Teor. Fiz. 126, 650 (2004) [JETP 99, 566 (2004)].

22. A. I. Likhtenshteĭn, A. S. Moskvin, and V. A. Gubanov, Fiz. Tverd. Tela (Leningrad) 24, 3596 (1982) [Sov. Phys. Solid State 24, 2049 (1982)].

23. A. V. Postnikov, St. Bartkowski, M. Neumann, et al., Phys. Rev. B 50, 14849 (1994).

24. V. V. Zhdanova, private communication.

25. D. P. Belanger, A. R. King, and V. Jaccarino, Phys. Rev. B 29, 2636 (1984).

Translated by A. Bagatur'yants 